Cahiers d'études italiennes

\title{
L'image de la femme dans l'œuvre de Primo Levi : une séduction ambiguë
}

Sophie Nezri-Dufour

\section{OpenEdition}

\section{Journals}

Édition électronique

URL : http://journals.openedition.org/cei/831

DOI : $10.4000 /$ cei.831

ISSN : 2260-779X

Éditeur

UGA Éditions/Université Grenoble Alpes

\section{Édition imprimée}

Date de publication : 15 septembre 2006

Pagination : 185-194

ISBN : 978-2-84310-086-4

ISSN : 1770-9571

\section{Référence électronique}

Sophie Nezri-Dufour, « L'image de la femme dans l'œuvre de Primo Levi : une séduction ambiguë », Cahiers d'études italiennes [En ligne], 5 | 2006, mis en ligne le 15 mars 2008, consulté le 26 mars 2021. URL : http://journals.openedition.org/cei/831 ; DOI : https://doi.org/10.4000/cei.831 


\title{
L'IMAGE DE LA FEMME DANS L'CEUVRE DE PRIMO LEVI
}

\author{
UNE SÉDUCTION AMBIGUË
}

\author{
Sophie Nezri-Dufour \\ Université de Provence - Aix-Marseille 1
}

Chez Primo Levi, la femme offre l'image d'une séductrice dont les agissements ont une implication très forte aussi bien dans la vie de l'ex-déporté que dans l'ensemble de son œuvre. Miroir d'une société en pleine mutation, touchée de plein fouet par la Shoah, la femme renvoie, dans des romans comme Il sistema periodico (publié en 1975) ou Se non ora, quando? (publié en 1982), à l'image d'une séduction symbole de paradis perdu et de réconfort, mais aussi, parallèlement, par sa dimension à la fois sexuelle et sociale, de désordre et de totale remise en question. Le terme «séduction» n'est-il pas d'ailleurs, étymologiquement, synonyme de séparation, de divergence?

La séduction devient alors, chez Levi, étroitement liée à l'idée d'altérité. Une altérité tout aussi ambivalente, car elle renferme à la fois les rêves et les peurs les plus inavoués de l'écrivain.

La séduction est donc toujours marquée, chez l'auteur turinois, par une Histoire apocalyptique et par les blessures d'un individu que l'on a toujours considéré, à tort, comme un pur esprit, rationnel, sans tenir compte de sa fragilité et de ses doutes ${ }^{1}$.

Dès lors que Primo Levi entre dans l'âge adulte, il est en effet intéressant de voir que ses rapports problématiques et souvent décevants avec les femmes coïncident avec ses rapports difficiles et désespérants avec l'Histoire, au moment même où s'organise au niveau de l'État la discrimination des juifs en Italie.

1. C'est ce que j'ai tenté de démontrer dans mon essai: Nezri-Dufour S., Primo Levi, una memoria ebraica del Novecento, Florence, la Giuntina, 2002. 
On se rend compte à quel point le jeu de la séduction devient alors, pour le jeune Turinois, un enjeu vital. En 1938, Primo Levi a en effet 19 ans, il entre à l'Université, et commence à s'intéresser aux filles. Mais la difficulté qu'il rencontre à les aborder semble aller de pair avec l'exclusion que le régime fasciste semble vouloir lui faire subir, avec la proclamation des lois raciales. Pourtant, son désir le plus fort, à l'époque, est d'être aimé d'une femme, sans doute pour s'assurer qu'il fait toujours partie d'une société qui l'isole peu à peu. Ainsi écrit-il, dans Il sistema periodico: «Mi sentivo frustrato e sfidato. Mi sentivo disperato, anzi [...] in quel tempo mi credevo condannato ad una perpetua solitudine mascolina, negato per sempre al sorriso di una donna, di cui pure avevo bisogno come dell'aria ${ }^{2}$.

Son origine juive, condamnée par le régime, est ainsi source d'un complexe qu'il conservera longtemps, d'autant plus qu'à l'époque, ses camarades non juifs insistent volontiers sur le fait que sa circoncision ne serait finalement rien d'autre qu'une castration. La différence intrinsèque entre un homme et une femme se double alors de cette différence identitaire - à la fois religieuse et culturelle - qui envahit et trouble profondément son existence: "Perché ebreo sono [...] e lei no", écrit-il à propos d'une jeune fille qu'il courtise, «sono io l'impurezza [...], sono il granello di sale e di senape. L'impurezza, certo» (p. 460).

Et lorsqu'il va jusqu'à oser passer son bras sous celui de cette même jeune fille, qu'il raccompagne chez elle, ce n'est pas seulement une victoire sur sa timidité sexuelle qu'il remporte, mais également une première victoire contre une Histoire hostile, qui semble lui refuser le bonheur et la normalité. Il racontera à ce sujet:

Tremando per l'emozione, infilai il mio braccio sotto il suo. Rita non si sottrasse, e neppure ricambiò la stretta: ma io regolai il mio passo sul suo, e mi sentivo ilare e vittorioso. Mi pareva di aver vinto una battaglia, piccola ma decisiva contro il buio, il vuoto, e gli anni nemici che sopravvenivano. (p. 461)

La séduction joue donc à cette époque, pour Primo Levi, un rôle essentiel, car elle a une valeur presque historique. Pour lui, jeune juif mis au ban de la société, il ne s'agit pas, lorsqu'il courtise des jeunes filles, de simple badinage amoureux, mais d'une véritable bataille contre un destin et une société qui lui sont hostiles. S'il réussit, lui l'exclu, à se faire aimer, et à séduire, c'est qu'il a finalement une place dans cette société, et une 
chance de survivre socialement. Par contre, si même la femme le repousse, c'est que son existence sociale lui est définitivement niée, refusée. Sa peur, bien légitime, est ainsi de voir sa propre existence remise en cause, à la fois par le régime, qui stigmatise et refuse sa judéité, et par la femme, qui pourrait l'éviter, le repousser.

Ainsi, lorsque, quelque temps plus tard, une collègue de travail cherche à lui présenter des jeunes filles, Primo Levi comprend bien que «la sua fretta di liberarmi di una vecchia angoscia, e di procurarmi subito una modesta porzione di gioia, veni[va] da una sua intuizione oscura di quanto il destino mi stava preparando, e mira[va] inconsapevolmente a deviarlo» (p. 537).

On voit là encore à quel point les rapports de Primo Levi avec les femmes sont intimement liés à l'Histoire, à une Histoire qui semble lui refuser sa part du bonheur. Il a pour ennemies non seulement les lois raciales, mais aussi, en fin de compte, les femmes, chez qui il ne parvient pas à trouver le réconfort dont il a tant besoin. Ces dernières, séductrices avant tout, occupent alors dans sa vie une place essentielle, mais problématique, puisqu'elles participent des temps difficiles qu'il est en train d'expérimenter. Ainsi écrivait-il, évoquant l'un de ses amours de jeunesse:

Venne un furioso temporale, Giulia resistette a due fulmini, al terzo cercò rifugio contro di me. Sentivo il calore del suo corpo contro il mio, vertiginoso e nuovo, noto nei sogni, ma non restituii l'abbraccio [car Giulia est fiancée, et lui-même ne croit pas au bonheur] ; se lo avessi fatto, forse il suo destino e il mio sarebbero usciti fragorosamente dai binari, verso un comune avvenire totalmente imprevedibile. (p. 538)

Femmes et lois raciales se coalisent donc pour lui refuser la possibilité de goûter à la vie, avec légèreté et insouciance, comme n'importe quel jeune Italien de son âge. Evoquant son inhibition sexuelle, il écrira, sur un ton tragi-comique: "La mia incapacità di avvicinare una donna era una condanna senza appello, che mi avrebbe accompagnato fino alla morte, restringendomi ad una vita avvelenata dalle invidie e dai desideri astratti, sterile e senza scopo" (p. 544).

Et lorsque le jeune Turinois raccompagne celle qu'il aime sur son vélo, pour qu'elle puisse rejoindre plus vite son fiancé, un bel "aryen" insouciant, c'est avec une grande amertume qu'il expérimente le lien étroit entre une Histoire injuste et ses rapports difficiles aux femmes:

Mi sentivo crescere dentro, forse per la prima volta, una nauseabonda sensazione di vuoto: questo, dunque, voleva dire essere altri, questo il prezzo di essere il sale della terra. Portare in canna una ragazza che si desidera, ed esserne talmente lontani da non potersene neppure innamorare: portarla in canna in Viale Gorizia per aiutarla ad essere di un altro, ed a sparire dalla mia vita. (p. 544) 
La femme devient ainsi synonyme de cette vie, de ce bonheur, de cette insouciance qui lui sont refusés durant les lois raciales. Et s'étant refusée à lui, la femme devient alors source de solitude et de frustration. D'où, dans son œuvre comme dans sa pensée, une conception tourmentée de la séduction et de l'amour, où la femme s'apparente à l'Autre, souvent ennemi, en tout cas problématique. Et par là même, la femme devient, tout comme l'Histoire, une cruelle initiatrice à l'âge adulte. Elle contribue à sortir Primo Levi de l'enfance et de ses illusions.

Il faudra attendre l'arrivée de Lucia, son épouse, pour qu'il se réconcilie avec les femmes, et parallèlement avec la vie. La rencontre avec cette jeune fille juive turinoise, aux lendemains d'Auschwitz, correspondra à un véritable cadeau du destin qui, jusque-là, lui avait été plus qu'hostile. Toujours dans Il sistema periodico, il écrira:

Avvenne che [...] il destino mi riserbasse un dono diverso e unico : l'incontro di una donna, giovane e di carne e d'ossa, calda contro il mio fianco attraverso i cappotti, allegra in mezzo alla nebbia [...], paziente sapiente e sicura mentre camminavamo per le strade ancora fiancheggiate di macerie [...]. In poche ore mi ero sentito nuovo e pieno di potenze nuove, lavato e guarito dal lungo male, pronto finalemente ad entrare nella vita con gioia e vigore, ed esorcizzato il nome e il viso della donna che era discesa agli inferi con me e non ne era tornata. (p. 572)

Il ne faudra en effet jamais oublier la présence fantomatique d'une jeune femme qu'il aima, Vanda Maestro, compagne de résistance arrêtée et déportée en même temps que lui, puis gazée à Auschwitz, qui reviendra plus d'une fois hanter ses pensées et ses pages, comme nous aurons l'occasion de le voir. Là encore, femme et Histoire seront intimement liées, puisque Vanda Maestro deviendra le paradigme d'une humanité innocente, et d'une humanité féminine, disparue dans les camps.

Mais grâce à Lucia, l'instinct de vie reprendra le dessus. Cette femme, dans sa séduction la plus positive ("giovane», "calda», "allegra», "paziente sapiente e sicura»), s'érigera alors comme emblème vital d'optimisme, tirant Primo Levi hors de l'univers de la mort. Même si la seconde existence de l'écrivain turinois prendra racine sur les ruines laissées par la guerre et le massacre nazi, c'est par l'intermédiaire d'une femme qu'il se réconciliera, du moins en apparence, et dans un premier temps, avec l'existence.

Dès lors, la femme conservera toujours deux visages dans l'œuvre de Primo Levi: elle sera tantôt l'ennemie, liée à la cruauté de la vie, de l'Histoire, à l'âge adulte aussi, et donc à une prise de conscience de soimême, de ses faiblesses, de ses peurs, et de ses fantasmes; tantôt la femme idéale renvoyant à un paradis, perdu le plus souvent, à une enfance 
humaine, à une femme-mère mais aussi à un alter ego symbiotique, ce deuxième spécimen étant le plus souvent lié à un rêve utopique.

On le voit bien dans le roman Se non ora, quando?, où plusieurs femmes, appartenant au passé, revêtent une dimension idéale mais malheureusement irréelle ou dépassée. Ainsi en est-il de Rivke, la femme de Mendel, qui est le protagoniste du roman, et qui ressemble, sous bien des aspects, à Primo Levi. Mendel est un résistant juif qui, entouré de ses compagnons, traverse l'Europe en flammes et combat les nazis. Rivke est la femme qu'il a perdue, qui a été assassinée et jetée dans une fosse commune. Son souvenir hante Mendel, totalement déstructuré depuis la mort de sa femme, dont la séduction résidait dans des valeurs simples qui désormais n'ont plus cours:

[Rivke] apparteneva all'ordine, al mondo delle cose giuste fatte alle ore giuste [...]. Mi faceva coraggio quando ne avevo bisogno: $\mathrm{mi}$ ha fatto coraggio perfino il giorno che è scoppiata la guerra e io sono partito per il fronte [...]. Eravamo una carne [...] era $[\ldots]$ una regina della casa ${ }^{3}$.

Dans sa séduction la plus pure et la plus innocente, Rivke appartient à un univers englouti, révolu, détruit par la Shoah, dont il est nécessaire de s'extraire pour aller de l'avant: un univers devenu idéal depuis qu'il n'existe plus, un univers de la mémoire où la séduction de la femme est idéalisée par la nostalgie; un univers où la séduction n'est pas synonyme de coquetterie ou de mensonge, mais de stabilité, d'équilibre et de sérénité. Or, l'Histoire a bouleversé et anéanti cet univers, et a détruit à jamais cette image de la femme, et surtout du couple édénique qu'elle continuait à perpétuer: "Eravamo una carne» est en effet une référence directe au couple primordial d'Adam et Eve (Genèse, 2, 24), avant que les choses ne se gâtent: "Senza Rivke, se dit Mendel, saresti un altro uomo, che pensa chissà come, un non-Mendel. Senza Rivke, senza l'ombra di Rivke, saresti pronto per l'avvenire. Pronto a vivere, a crescere come un seme» (p. 485).

Dans l'esprit de Mendel, alter ego romanesque de Primo Levi, la femme idéale n'existe donc plus, tout comme le passé de son enfance, qui lui aussi a été anéanti. Son épouse assassinée est l'emblème des victimes de la Shoah, et de l'impossibilité d'un avenir paisible et normal. On voit que, là encore, femme et Histoire sont intimement liées, puisque la Shoah, par le Chaos et l'horreur qu'elle a engendrés, a totalement bouleversé les rapports humains et le statut des individus, notamment celui de la femme

3. Id., Se non ora, quando?, Opere II, Turin, Einaudi, «Bilioteca dell'Orsa», 1988, p. 426. Dorénavant, l'indication des pages sera donnée directement après la citation, entre parenthèses. 
dans la société. On ne peut désormais plus penser et appréhender les choses comme avant: Auschwitz oblige à penser autrement. Ainsi, si Mendel cherche en Sissl, une résistante qui se bat à ses côtés, le réconfort de la femme idéale, douce, maternelle, il sait en même temps que l'apparente sérénité qu'elle lui apporte n'est qu'éphémère. Néanmoins, les moments d'amour qu'ils vivent sont le lointain écho d'un rapport rempli d'harmonie et de douceur: "Faceva freddo, si spogliarono a mezzo, e Mendel fu stordito dall'odore femmineo di Sissl e dal bagliore della sua pelle. Sissl si aprì come un fiore, docile e calda; Mendel si sentì irrompere nelle reni la forza e il desiderio che da due anni tacevano» (p. 297).

Dans ce contexte, la séduction féminine réside dans la vie et l'énergie que la femme parvient à insuffler à l'homme. Elle symbolise la douceur, la sécurité, la paix, un goût de paradis: "Non ci fu bisogno di parole, fu naturale e dovuto come nel Paradiso Terrestre» (p. 296).

Mais en même temps, cette Sissl, douce, réservée, proche de la mythique Rivke, reste paralysée dans son rôle de femme traditionnelle, et ne parvient pas à offrir à Mendel de nouveaux horizons dans un univers qui n'est plus celui d'autrefois ${ }^{4}$.

Line, au contraire, autre personnage de Se non ora, quando?, est une résistante juive, féministe, qui représente la femme de l'après-Shoah, et revêt une séduction qui fascine et, dans le même temps, effraie Mendel. La séduction qu'elle dégage est celle de la femme qui a appris à survivre aux atrocités de l'Histoire, et qui revendique l'égalité des sexes, luttant dans un monde encore extrêmement violent, agressif, puisque la guerre n'est pas finie. Consciente que le passé juif d'Europe de l'Est est désormais englouti, elle combat pour une nouvelle place de la femme dans une société masculine qui longtemps l'a reléguée et rejetée: "Le donne oggi vanno in esilio come gli uomini, explique-t-elle. Sono impiccate come gli uomini, e sparano meglio degli uomini» (p. 354-355).

Lorsqu'elle deviendra la maîtresse de Mendel, leur rapport amoureux sera bien différent de celui qu'avait eu Mendel avec Sissl. Line n'est la femme de personne, et n'a aucunement l'intention d'être liée et dominée. Sa séduction est celle d'une guerrière:

Mendel si spogliò, e subito Line gli si avvinghiò addosso come per una lotta. Schiacciata sotto il peso del corpo mascolino, Line si torceva, avversario tenace e resi-

4. Dans cette analyse, je m'inspire du travail de mon étudiante de maitrise, Sabrina Noémie, auteur de La rappresentazione della donna nell'opera di Primo Levi, Université de Provence, 20012002, $150 \mathrm{p}$. 
liente, per eccitarlo e sfidarlo. Era un linguaggio [...] : ti voglio ma ti resisto. Ti resisto perché ti voglio. Io esile ti giaccio sotto ma non sono tua. Io non sono la donna di nessuno; e resistendo ti lego a me. Mendel la sentiva armata anche nuda. (p. 361)

Line est la femme moderne qui a su se battre et se construire seule, malgré l'adversité, et sans l'aide des hommes. Indépendante, forte, courageuse, sa séduction naît essentiellement de ses qualités morales et intellectuelles, de sa potentialité à être sûre d'elle, à ne pas douter, afin de mieux survivre. Loin de la séductrice conventionnelle, elle est décrite en des termes qui font d'elle une femme nouvelle, volontaire, qui brise toutes les images de la séduction féminine traditionnelle. Elle a su s'adapter aux métamorphoses de l'Histoire, en se métamorphosant elle-même, et en s'imposant dans un univers essentiellement masculin:

Era una partigiana, non una rifugiata. Mendel aveva ammirato [...] la sua destrezza nel maneggiare le armi, e durante la marcia sulla neve la sua resistenza [...]. Questo non è un dono della natura, pensava : è una riserva di coraggio e di forza che va ricostituita ogni giorno, dovremmo fare tutti come lei. Questa ragazza sa volere [...]. Line parlava poco, e mai inutilmente [...]. Aveva modi diversi da quelli delle donne, ebree e non, che Mendel aveva incontrato fino allora. Non mostrava ritrosie né falsi pudori, non recitava e non faceva capricci. (p. 297-298)

La femme doit désormais se transformer, se métamorphoser, afin de s'adapter aux soubresauts de l'Histoire. Première à se rendre compte de la nécessité de s'adapter au nouveau contexte historique qui se met en place, elle offre une image d'elle-même en mouvement perpétuel, jamais définitive. Plus que ses compagnons, elle représente l'Homme moderne.

Hybride, elle a désormais plusieurs fonctions, plusieurs vertus qui fascinent mais inquiètent. Reflet du changement de l'époque, elle offre aux hommes le miroir d'une société en pleine mutation, difficile à saisir. C'est ce qui séduit Mendel mais en même temps le perturbe: «Davanti a Line, Mendel non poteva sottrarsi all'impressione di una sostanza umana preziosa ed insolita ma inquieta ed inquietante. Sissl era come una palma al sole, Line era un'edera intricata e notturna» (p. 297).

Line est cette femme qui lie mais ne se lie pas, comme si elle envahissait l'esprit de celui qui est lié à elle pour l'aliéner. Elle personnifie donc également une séduction dérangeante, trop moderne, et trop nouvelle pour ne pas déstabiliser. De même que l'époque que Mendel est en train de vivre remet en question tous ses repères, de la même manière, une femme telle que Line remet en question toute une partie de ses convictions et de ses certitudes. Elle le met face à la Réalité historique, nouvelle et perturbante. 
Line n'en est que plus séduisante mais elle lui fait peur. Réveillant en lui toute une série de fantasmes, elle réveille ses angoisses et son irrationalité, liées à la fois à la sexualité et au chaos historique:

A Mendel, quando osservava Line, veniva in mente Raab, la seduttrice di Gerico, e le altre ammaliatrici della leggenda talmudica [...]. Michàl, che affascinava chi la vedeva. Giaele, la mortifera partigiana di un tempo, che aveva trafitto le tempie del generale nemico con un chiodo, ma che seduceva tutti gli uomini col suono della sua voce. Abigail, la regina assennata, che seduceva chiunque pensasse a lei. Ma Raab era superiore a tutte, qualsiasi uomo pronunciasse soltanto il suo nome spandeva istantaneamente il suo seme. (p. 298)

On voit par cet exemple que pour Mendel-Levi, la femme est celle qui, dans sa séduction, brise l'équilibre et provoque le désordre dans l'esprit de l'homme. Elle représente cet irrationnel que craint tant Primo Levi, ce côté inconscient que l'homme ne peut pas toujours maîtriser, et qui fait de lui un individu sous l'emprise de pulsions, de fantasmes, et d'angoisses qui le dépassent. Elle est l'incompréhensible, attirant, mais qui entraîne l'homme dans ce qu'il ne domine plus. Elle est la matière, fuyante, qui s'oppose et nuit à l'Esprit.

En outre, à l'inverse de Rivke qui ne formait qu'une seule chair avec Mendel, et qui représentait donc l'Eve de l'Eden, créée directement à partir d'Adam, Line, de son côté, représente Lilith, personnage omniprésent dans l'œuvre de Primo Levi ${ }^{5}$, qui selon certains textes de la tradition juive (le Zohar), aurait été la première femme d'Adam, créée indépendamment de celui-ci, contrairement à Eve. Féministe avant l'heure, Lilith, aspirant à être l'égale de l'homme, aurait refusé, au moment de l'acte d'amour, de se coucher sous Adam, et se serait par la suite enfuie, devenant une diablesse (Zohar II, 22 b).

Line est la Lilith de Primo Levi et de Mendel: elle aussi refuse d'être dominée durant l'acte d'amour, elle aussi abandonne Mendel pour ne pas se lier. Elle est le nouveau type de femme que la Shoah a créé, et dont le rôle s'est élargi. Elle ne forme plus une entité indissociable et harmonieuse avec son partenaire. Elle n'est plus, systématiquement, aux côtés de l'homme, mais en face de lui. Elle lui renvoie alors sa propre image, l'image d'un individu qui a perdu ses repères traditionnels, et qui ne mâ̂trise plus sa destinée.

5. On pensera notamment au poème "Lilith" in Ad ora incerta in Opere II, op. cit., p. 547, ou au recueil de nouvelles intitulé Lilit, Opere III, Torino, Einaudi, «Biblioteca dell'Orsa», 1990, p. 373-577. 
Cette peur-fascination est celle d'un Primo Levi qui, depuis le début, nous l'avons vu, nourrit un sentiment ambivalent vis-à-vis de la séduction féminine. Pour lui, la femme est à la fois fascinante et incompréhensible, car elle offre la nouveauté, le plaisir, mais aussi le désordre, l'incertitude. Elle est vitalité et absence, plénitude et vide. Elle représente un bonheur concret, physique, tangible, mais elle s'apparente du même coup à la matière, et donc à une nature qui éloigne l'homme de sa raison et de sa stabilité.

Représentant la séduction par essence, la femme s'associe en effet au sexe, donc à l'instinct, à la fatalité d'une nature que l'on ne peut pas contrôler, et c'est ce qui fait peur à Primo Levi. Soucieux d'être toujours maitre de ses émotions, de ses pensées, il sait que la femme peut susciter en l'homme une avalanches d'émotions mal maîtrisées, une violence qui le répugne, un vice de l'esprit qui l'affaiblisse, et le diminue intellectuellement.

Par sa nouvelle dimension historique, et par sa charge sexuelle très forte, la femme revêt alors la dimension paradigmatique de l'Autre, car elle remet l'homme en question, et lui interdit toutes certitudes, toute sécurité, tout projet assuré. Mais par là même, elle lui permet aussi d'évoluer, de remettre en question des notions figées. Elle est un miroir, nous l'avons vu, qui peut devenir le miroir des questionnements enfouis de l'homme. Elle le sort de ses convictions, pour lui révéler l'inconnu, souvent effrayant, et donc le faire pénétrer dans son propre monde, et dans le monde adulte. Belle et monstrueuse, douce et cruelle dans le même temps, elle est l'image de l'existence, mystérieuse et imprévue, mais qu'il faut savoir affronter dans ses métamorphoses. Les certitudes du passé ont été détruites à Auschwitz, et la métamorphose nécessaire de la femme en est un reflet. D'où une profonde nostalgie pour ce qui ne reviendra plus, car l'homme aussi va devoir changer.

Symbole d'altérité, la femme devient alors le catalyseur d'une peur de la complexité des choses qui se transforment et peuvent disparaître. Elle est souffle divin et poussière, elle représente donc cette dichotomie inhérente à la nature humaine, faite de boue et d'esprit, qui trouble tant notre auteur turinois, si méfiant, à cause d'Auschwitz, à l'égard des passions humaines.

Créature à la fois concrète et abstraite, presque mythique, vitale et nocive, elle ouvre à un univers qui refuse toute définition figée, sclérosée. Et ce qu'elle a gagné, elle, en confiance et en courage par la force des choses, elle semble l'avoir retiré à l'homme, qui désormais doute plus que 
jamais. "E Line? Che dire di Line?», pense Mendel à la fin du roman, lorsque la guerre est finie:

È una madre-moglie-figlia-amica-nemica-rivale-maestra. È stata carne della mia carne, io sono entrato in lei, mille anni fa [...] quando c'era ancora la guerra e il mondo era giovane e ognuno di noi era un angelo con la spada in mano [...]. Eccola accanto a me, non guarda me ma guarda fisso questo paesaggio tedesco e sa sempre con esattezza quello che si deve fare. Mille anni fa, nelle paludi, lo sapevo anch'io e adesso lei lo sa ancora e io non lo so più [...]. Ma quando rivedo il suo corpo nella memoria, quando lo indovino sotto le vesti, mi sento lacerare, e vorrei ricominciare, e so che non si può e proprio per questo mi sento lacerare. (p. 485) 